\title{
Relativistic Stellar Clusters: Equilibrium Models with Anisotropic Momentum Distribution and Dynamic and Thermodynamic Stability of Isotropic Models
}

\author{
Marco Merafina*, Gennady S. Bisnovatyi-Kogan ${ }^{\dagger}$ and Maria Rosaria \\ Vaccarelli* \\ *Department of Physics, University of Rome "La Sapienza" Piazzale Aldo Moro 2, I-00185 Rome, \\ Italy \\ ${ }^{\dagger}$ Space Research Institute (IKI) Profsoyuznaya 84/32, Moscow 117997, Russia
}

\begin{abstract}
Models of spherically symmetric relativistic stellar clusters with anisotropic distribution functions in relativistic regime are described by using Maxwellian distribution function with energy cutoff. We consider distributions with different levels of anisotropy and discuss some general characteristics of the models. In addition, we analyze dynamic and thermodynamic stability of isotropic models still described by Maxwellian distribution function with energy cutoff and we find critical values of the onset of instability.
\end{abstract}

Keywords: Relativity - Stellar Clusters - Anisotropy - Stability

PACS: $04.20 .-\mathrm{q}, 98.20 .-\mathrm{d}$

\section{INTRODUCTION}

One of the possible ways of formation of supermassive black holes in quasars and galactic nuclei is the collapse of a dense stellar cluster after loss of dynamic stability. Starting from the pioneering work of Zel'dovich \& Podurets [24], the problem of the structure and stability of relativistic stellar clusters (RSC) was studied by many authors.

In the papers of Ipser [14, 15], Suffern \& Fackerell [23] and, more recently, in the works of Bisnovatyi-Kogan et al. [3, 4], thereafter named BKMRV93 and BKMRV98 respectively (see also references therein), the structure and stability of RSC was studied by using the solution of general relativistic non-collisional Boltzmann equation (RnCBE), similar to what introduced in the paper of Zel'dovich \& Podurets. Numerical simulations of RSC consisting of non-collisional point masses in a common gravitational field had been performed by Rasio et al. [21].

Numerical calculations of the evolution of Newtonian stellar clusters due to close encounters among stars are performed by solving directly non-relativistic Fokker-Planck equation or using Monte-Carlo simulations (see Gürkan et al. [12] and references therein). The qualitative picture of the evolution of dense stellar clusters of different masses taking into account quite different physical processes had been analyzed by Bisnovatyi-Kogan [2]: it was shown that, due to star evaporation from the cluster during its evolution, the mass of relativistically collapsing core does not exceed approximately $0.1 \%$ of its whole initial mass. 
In most papers, mentioned above, where solutions of RnCBE had been obtained, isotropic distribution functions $f(E)$ had been considered. Nevertheless, already Einstein [11] had constructed a highly relativistic cluster with circular orbits. Analytic self-similar solutions for RSC with arbitrary level of anisotropy had been found by Bisnovatyi-Kogan \& Zel'dovich [8,9]: in the former paper all solutions formally had infinite central densities and infinite radii, so they could not be applied directly to the reality.

It is also important to mention here papers with solutions for anisotropic stellar clusters obtained in Newtonian approximation (Bisnovatyi-Kogan \& Zel'dovich [9]; Ingrosso et al. [13]). Being a large part of matter in the Universe consisting of "dark" non-collisional component, we can argue that it forms equilibrium configurations well described by non-collisional Boltzmann equation. Evolution of dark matter clusters and formation of a large scale structure can lead to formation of systems with an arbitrary level of anisotropy which could be described by distribution functions far from equilibrium isotropic Maxwell-Boltzmann one.

Anisotropic stellar clusters with larger transverse momentum (the extreme case is a cluster with purely circular orbits) are more stable to relativistic collapse than the isotropic clusters and loose stability in general relativistic regime at larger mass concentration. Anisotropy in the momentum space appears always on the stage of a rapid contraction of the cluster due to preservation of the angular momentum. Strong anisotropy in the momentum distribution is expected in dense clusters with a supermassive black hole in the center, where in the vicinity of the last stable orbit only stars with circular orbits can survive.

Here, we consider relativistic models with anisotropic distribution in the momentum space. We use the same approach for obtaining the solutions as in BKMRV93, BKMRV98 and in Bisnovatyi-Kogan \& Merafina [5] and restrict ourself to analysis of equilibrium configurations. Finally, we investigate the stability of isotropic clusters, giving a brief analysis of the results.

\section{MAIN EQUATIONS}

Let us consider spherically symmetric equilibrium configurations with a Schwarzschildlike metric (see Landau \& Lifshitz [16])

$$
d s^{2}=e^{v} c^{2} d t^{2}-e^{\lambda} d r^{2}-r^{2}\left(d \theta^{2}+\sin ^{2} \theta d \varphi^{2}\right) .
$$

The exact solution of RnCBE must depend on integrals of motion which are represented by energy $E$ and angular momentum $L$, here expressed by the following relations

$$
E=e^{v / 2}\left(p^{2} c^{2}+m^{2} c^{4}\right)^{1 / 2} \quad \text { and } \quad L=p_{t} r
$$

where $m$ is the stellar mass and $p=\sqrt{p_{r}^{2}+p_{t}^{2}}$ is the stellar momentum, being $p_{r}$ and $p_{t}$ the radial and transversal components, respectively. We search a solution depending only on $E$ and $L$ in the form (Bisnovatyi-Kogan et al. [6, 7]) 


$$
\begin{gathered}
f=A\left(1+\frac{L^{2}}{L_{c}^{2}}\right)^{l} e^{-E / T} \text { for } E \leq E_{c}, \\
f=0 \text { for } E>E_{c},
\end{gathered}
$$

where $T$ (constant) is the temperature in energy units measured by an infinitely remote observer and $L_{c}=m c r_{a}$ is a constant depending on anisotropy radius $r_{a}$ that represents the value of the radius beyond which the orbits begin to be tangential in prevalence. The cutoff energy is chosen, following Zel'dovich \& Podurets [24], in the form

$$
E_{c}=m c^{2}-\alpha T / 2 .
$$

In order to find the equilibrium configurations representing different models of RSC, we solve the relativistic equilibrium equations (see e.g. Bisnovatyi-Kogan \& Zel'dovich [8]) which generalize the well known Oppenheimer-Volkoff equations in presence of an anisotropic pressure tensor with $P_{r r} \neq P_{t}$. The equations are

$$
\begin{gathered}
\frac{d P_{r r}}{d r}=-\frac{G}{r c^{2}} \frac{\left(P_{r r}+\rho c^{2}\right)\left(M_{r} c^{2}+4 \pi P_{r r} r^{3}\right)}{r c^{2}-2 G M_{r}}-\frac{2}{r}\left(P_{r r}-P_{t}\right), \\
\frac{d M_{r}}{d r}=4 \pi r^{2} \rho,
\end{gathered}
$$

with boundary conditions $P_{r r}(0)=P_{0}$ and $M_{r}(0)=0$. The metric coefficients are determined by relations

$$
\begin{gathered}
e^{\lambda}=\left(1-\frac{2 G M_{r}}{r c^{2}}\right)^{-1}, \\
\frac{d v}{d r}=\frac{2 G}{c^{2}} \frac{M_{r} c^{2}+4 \pi P_{r r} r^{3}}{r\left(r c^{2}-2 G M_{r}\right)} \\
e^{\nu_{R}}=e^{-\lambda_{R}}=1-\frac{2 G M}{R c^{2}}
\end{gathered}
$$

where $M_{r}$ is the mass inside a given Lagrangian radius $r$. The solutions of equilibrium equations describing RSC have density and both components of pressure vanishing at the outer boundary $r=R$.

\section{THERMODYNAMIC QUANTITIES}

Thermodynamic quantities as the concentration $n$, connected with the rest mass density $\rho_{0}=n m$, the total energy density $\rho c^{2}$ and the components of the stress tensor $\left(P_{r r}, P_{t}\right)$ are expressed by integrals containing the distribution function $f$ of Eq. (3). Introducing the angle $\theta$ in the plane $\left(p_{r}, p_{t}\right)$, so that

$$
p_{r}=p \cos \theta, \quad p_{t}=p \sin \theta, \quad \text { with } \quad 0 \leq \theta \leq \pi
$$


and taking into account that $L=r p_{t}=r p \sin \theta$ and the Newton binomial relation

$$
\left(1+\frac{L^{2}}{L_{c}^{2}}\right)^{l}=\sum_{k=0}^{l}\left(\begin{array}{l}
l \\
k
\end{array}\right)\left(\frac{L^{2}}{L_{c}^{2}}\right)^{k},
$$

we have

$$
\begin{gathered}
n=2 \pi A \sum_{k=0}^{l}\left(\begin{array}{l}
l \\
k
\end{array}\right)\left(\frac{r}{L_{c}}\right)^{2 k} \int_{0}^{\pi}(\sin \theta)^{2 k+1} d \theta \int_{0}^{p_{c}} p^{2 k+2} e^{-E / T} d p \\
\rho c^{2}=2 \pi A \sum_{k=0}^{l}\left(\begin{array}{l}
l \\
k
\end{array}\right)\left(\frac{r}{L_{c}}\right)^{2 k} \int_{0}^{\pi}(\sin \theta)^{2 k+1} d \theta \int_{0}^{p_{c}} p^{2 k+2}\left(p^{2} c^{2}+m^{2} c^{4}\right)^{1 / 2} e^{-E / T} d p \\
P_{r r}=2 \pi A c^{2} \sum_{k=0}^{l}\left(\begin{array}{l}
l \\
k
\end{array}\right)\left(\frac{r}{L_{c}}\right)^{2 k} \int_{0}^{\pi}(\sin \theta)^{2 k+1} \cos ^{2} \theta d \theta \\
\times \int_{0}^{p_{c}} p^{2 k+4}\left(p^{2} c^{2}+m^{2} c^{4}\right)^{-1 / 2} e^{-E / T} d p \\
P_{t}=\pi A c^{2} \sum_{k=0}^{l}\left(\begin{array}{l}
l \\
k
\end{array}\right)\left(\frac{r}{L_{c}}\right)^{2 k} \int_{0}^{\pi}(\sin \theta)^{2 k+3} d \theta \int_{0}^{p_{c}} p^{2 k+4}\left(p^{2} c^{2}+m^{2} c^{4}\right)^{-1 / 2} e^{-E / T} d p
\end{gathered}
$$

For solving equations (5)-(6) with thermodynamic functions (12) it is convenient to introduce the following variables (see Merafina \& Ruffini $[19,20]$ )

$$
\begin{gathered}
\varepsilon=\left(p^{2} c^{2}+m^{2} c^{4}\right)^{1 / 2}-m c^{2} \quad(\text { current "particle" kinetic energy }), \\
\varepsilon_{c}=\left(p_{c}^{2} c^{2}+m^{2} c^{4}\right)^{1 / 2}-m c^{2} \quad(\text { maximal kinetic energy at a given radius } r), \\
T_{r}=T e^{-v / 2} \quad(\text { local temperature at a given radius } r),
\end{gathered}
$$

$\beta=\frac{T_{R}}{m c^{2}} \quad$ (nondimensional temperature, in energy units, at the edge of the cluster),

$W=\frac{\varepsilon_{c}}{T_{r}} \quad$ (nondimensional ratio of maximal kinetic energy to the local temperature),

$$
W_{0}=\frac{\varepsilon_{c}(0)}{T_{0}} \quad \text { (the value of } \mathrm{W} \text { at the center of the cluster). }
$$

Using the above variables we obtain the following relations

$$
\begin{gathered}
\frac{E}{T}=\frac{\varepsilon+m c^{2}}{T_{r}}, \quad \frac{E_{c}}{T}=\frac{\varepsilon_{c}(R)+m c^{2}}{T_{R}}=\frac{m c^{2}}{T_{R}}=\frac{1}{\beta}, \\
E_{c}=m c^{2}-\alpha T / 2=m c^{2} e^{v_{R} / 2}=m c^{2}\left(1-\frac{2 G M}{R c^{2}}\right)^{1 / 2},
\end{gathered}
$$




$$
W \equiv \frac{\varepsilon_{c}}{T_{r}}=\frac{m c^{2}}{T_{R}}-\frac{m c^{2}}{T_{r}}=\frac{1}{\beta}-\frac{m c^{2}}{T_{r}}, \quad x=\frac{\varepsilon}{T_{r}},
$$

where we have taken into account that the ratio $E_{c} / T$ is a constant all over the cluster and the stellar velocities at the outer boundary are zero, so that $\varepsilon_{c}(R)=0$. In addition, from Eqs. (14) we have also

$$
e^{-E / T}=e^{-\left(\varepsilon+m c^{2}\right) / T_{r}}=e^{-(x-W+1 / \beta)}=e^{W-x} e^{-1 / \beta}
$$

and therefore, from considerations of statistical mechanics, we can define the constant $B=A e^{-1 / \beta}$. Finally, due to these transformations, we obtain the following form of the integrals of Eqs. (12)

$$
\begin{gathered}
n=\pi B \sum_{k=0}^{l}\left(\begin{array}{l}
l \\
k
\end{array}\right)(\sqrt{2} m c)^{2 k+3}\left(\frac{r}{L_{c}}\right)^{2 k} A_{k}\left(\frac{\beta}{1-\beta W}\right)^{k+3 / 2} \\
\times \int_{0}^{W} e^{W-x}\left(1+\frac{\beta x / 2}{1-\beta W}\right)^{k+1 / 2}\left(1+\frac{\beta x}{1-\beta W}\right) x^{k+1 / 2} d x, \\
\rho c^{2}=\pi B \sum_{k=0}^{l}\left(\begin{array}{l}
l \\
k
\end{array}\right) m c^{2}(\sqrt{2} m c)^{2 k+3}\left(\frac{r}{L_{c}}\right)^{2 k} A_{k}\left(\frac{\beta}{1-\beta W}\right)^{k+3 / 2} \\
\times \int_{0}^{W} e^{W-x}\left(1+\frac{\beta x / 2}{1-\beta W}\right)^{k+1 / 2}\left(1+\frac{\beta x}{1-\beta W}\right)^{2} x^{k+1 / 2} d x, \\
P_{r r}=\pi B \sum_{k=0}^{l}\left(\begin{array}{l}
l \\
k
\end{array}\right) 2 m c^{2}(\sqrt{2} m c)^{2 k+3}\left(\frac{r}{L_{c}}\right)^{2 k}\left(A_{k}-A_{k+1}\right)\left(\frac{\beta}{1-\beta W}\right)^{k+5 / 2} \\
\times \int_{0}^{W} e^{W-x}\left(1+\frac{\beta x / 2}{1-\beta W}\right)^{k+3 / 2} x^{k+3 / 2} d x, \\
P_{t}=\pi B \sum_{k=0}^{l}\left(\begin{array}{l}
l \\
k
\end{array}\right) m c^{2}(\sqrt{2} m c)^{2 k+3}\left(\frac{r}{L_{c}}\right)^{2 k} A_{k+1}\left(\frac{\beta}{1-\beta W}\right)^{k+5 / 2} \\
\times \int_{0}^{W-x}\left(1+\frac{\beta x / 2}{1-\beta W}\right)^{k+3 / 2} x^{k+3 / 2} d x .
\end{gathered}
$$

The coefficients $A_{k}$ are represented by integrals over the trigonometric functions, which lead to analytic expressions (Bronstein \& Semendyaev [10])

$$
A_{k} \equiv \int_{0}^{\pi}(\sin \theta)^{2 k+1} d \theta=2 \sum_{i=0}^{k}\left(\begin{array}{l}
k \\
i
\end{array}\right) \frac{(-1)^{i}}{2 i+1}, \quad\left(\begin{array}{l}
k \\
i
\end{array}\right)=\frac{k !}{i !(k-i) !}, \quad 0 !=1
$$

and 


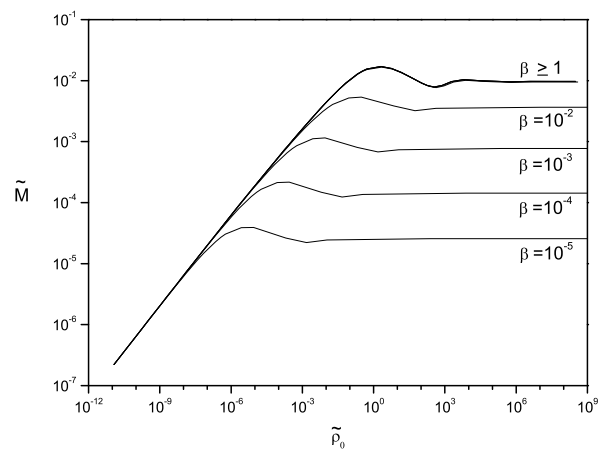

FIGURE 1. Mass of the cluster as a function of the central density at different values of $\beta$, for $l=1$ and $a=10^{-2}$. Increasing values of the central density correspond to larger values of the parameter $W_{0}$. The quantities are dimensionless.

$$
\int_{0}^{\pi}(\sin \theta)^{2 k+1} \cos ^{2} \theta d \theta=A_{k}-A_{k+1} .
$$

In particular, the first four values of $A_{k}$ are given by

$$
A_{0}=2, \quad A_{1}=\frac{4}{3}, \quad A_{2}=\frac{16}{15}, \quad A_{3}=\frac{32}{35} .
$$

In order to quantitatively evaluate the effects of anisotropy on RSC, it may be useful to introduce the local anisotropy level, defined as $\eta=P_{r r} / P_{t}$ (see also Bisnovatyi-Kogan et al. [6,7]). Due to the form of the distribution function (3), the value of $\eta$ is equal to unity (isotropic function) in the center and at the edge of the cluster, like the Newtonian case. Except these two points, $\eta<1$ al lover the cluster, since we have considered only positive values of $l$.

\section{NUMERICAL RESULTS}

The calculations have been performed for values $l=1,2$ of the index of the distribution function (3). The physical implications on the choice of other values of $l$, also negative ones, will be considered in a future paper, in which the main characteristics of such models will be described.

In Figs. 1-4 we represent nondimensional mass of the cluster as a function of its central density, at different values of the temperature parameter $\beta$, for indexes $l=$ 1,2 and anisotropy parameters $a=10^{-2}, 10^{-5}$. Here $a$ is the dimensionless quantity corresponding to anisotropy radius $r_{a}$. In these figures it is simple to note that the effects of the presence of anisotropy (at increasing $l$ and/or decreasing $a$ ) manifest a general 


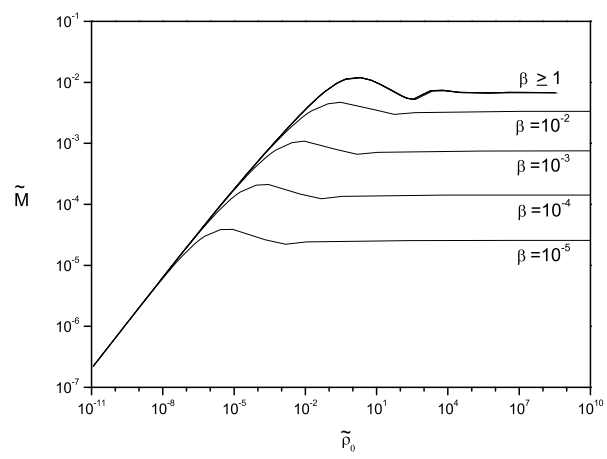

FIGURE 2. Same as in Fig. 1 for $l=2$ and $a=10^{-2}$.

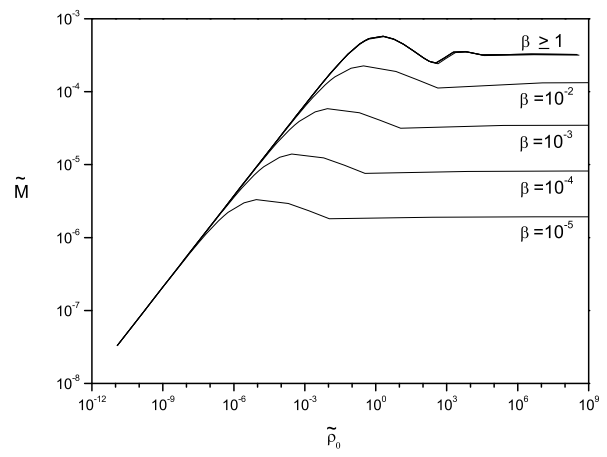

FIGURE 3. Same as in Fig. 1 for $l=1$ and $a=10^{-5}$.

decreasing of masses of the equilibrium configurations. We can also note that the curves converge for $\beta \geq 1$.

By considering a different point of view, in Fig. 5 we have represented the same curves described in the first four figures by varying the anisotropy parameter from $a=10^{-1}$ to $a=10^{-5}$, for fixed values $l=1$ and $\beta=1$. The effects of anisotropy on masses of equilibrium configurations previously indicated are well explicited; moreover, at smaller levels of anisotropy $(a \geq 0.1)$, the curves approach to the ones describing isotropic clusters, shown in the diagram (b) of Fig. 5 in BKMRV98. Note that, differently from the figure in BKMRV98, we have chosen to fix the parameter $\beta$ instead of $T$.

The level of the anisotropy in the distribution function (3) depends on the value of the parameter $a$. The local anisotropy level may be represented by the ratio between the 


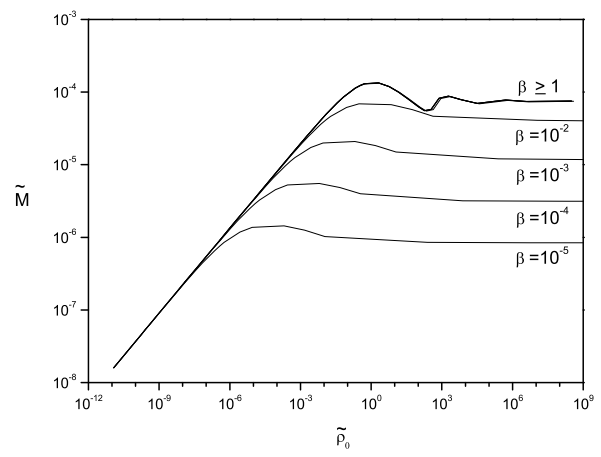

FIGURE 4. Same as in Fig. 1 for $l=2$ and $a=10^{-5}$.

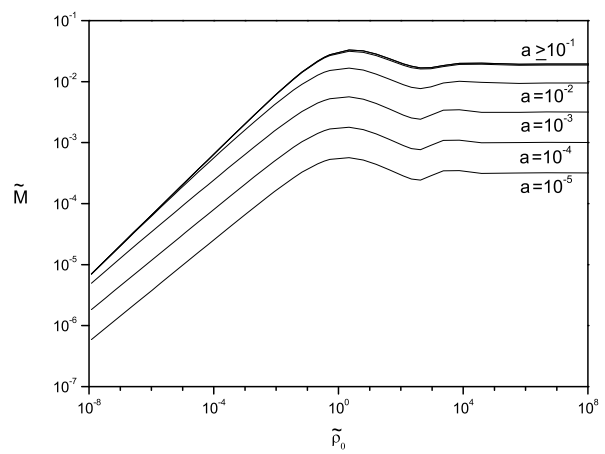

FIGURE 5. Mass of the cluster as a function of the central density at different values of $a$, for $l=1$ and $\beta=1$. The quantities are dimensionless.

components of the stress tensor (see Bisnovatyi-Kogan et al. [6, 7])

$$
\eta=\frac{2\left\langle v_{r}^{2}\right\rangle}{\left\langle v_{t}^{2}\right\rangle}=\frac{P_{r r}}{P_{t}} .
$$

The quantities $\left\langle v_{r}^{2}\right\rangle$ and $\left\langle v_{t}^{2}\right\rangle$ are the radial and tangential mean square velocity of stars, respectively. The value of $\eta$ approaches to unity for isotropic functions.

In Figs. 6-9 we have represented the quantity $\eta$ as a function of the relative radius $r / R$ for $l=1$ and selected values of the parameters $a, \beta$ and $W_{0}$. Due to the choice of a positive value of index $l$ in the distribution function (3), the anisotropy evidences a prevalence of tangential motion over the radial one, which is increasing with decreasing 


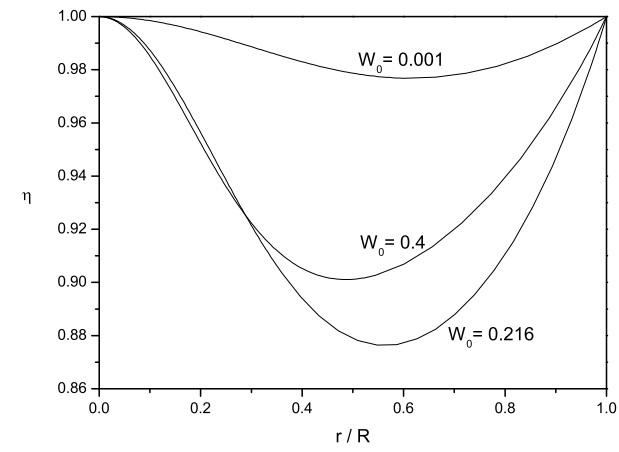

FIGURE 6. Values of the ratio of the pressure components $\eta=P_{r r} / P_{t}$ as a function of the relative radius $r / R$ along the cluster, for $l=1, a=10^{-1}, \beta=1$ and $W_{0}=0.001 ; 0.216 ; 0.4$.

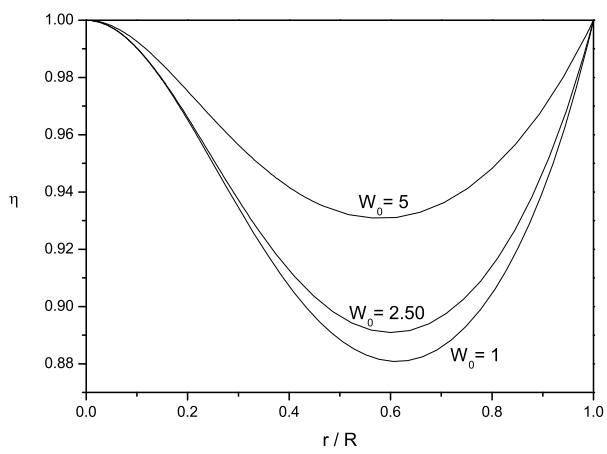

FIGURE 7. Same as in Fig. 6 , for $l=1, a=10^{-2}, \beta=10^{-5}$ and $W_{0}=1 ; 2.50 ; 5$.

of the parameter $a$. The triad of values of $W_{0}$ chosen in each figure refers to the equilibrium configuration which mass corresponds to the maximum value in $M$ vs $\rho_{0}$ diagrams of Figs. 1-5 and to values before and after this maximum.

It follows from the calculations that clusters described by the distribution (3) are isotropic not only in the center, but also at the edge of the configuration: in fact, it is easy to see from the expressions of the components of pressure (18) and (19) that $P_{r r} / P_{t} \rightarrow 1$ at the boundary of the cluster where $W \rightarrow 0$ and $r \rightarrow R$. As we may see in Figs. 6-9, the thickness of the external isotropic region is rapidly decreasing with decreasing of the anisotropy parameter $a$ which corresponds to higher levels of anisotropy. Moreover, we can express the quantity $\eta$ in the following way 


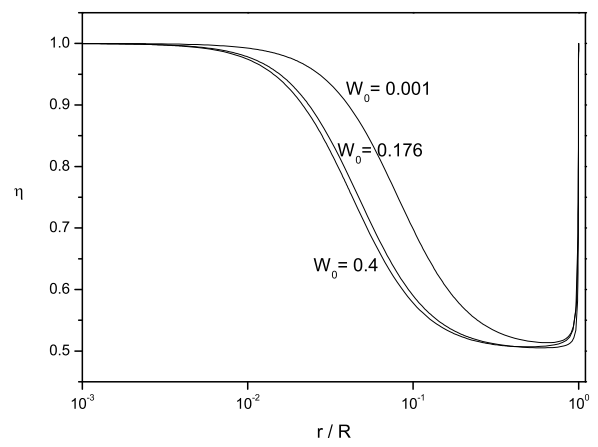

FIGURE 8. Same as in Fig. 6 , for $l=1, a=10^{-3}, \beta=1$ and $W_{0}=0.001 ; 0.176 ; 0.4$.

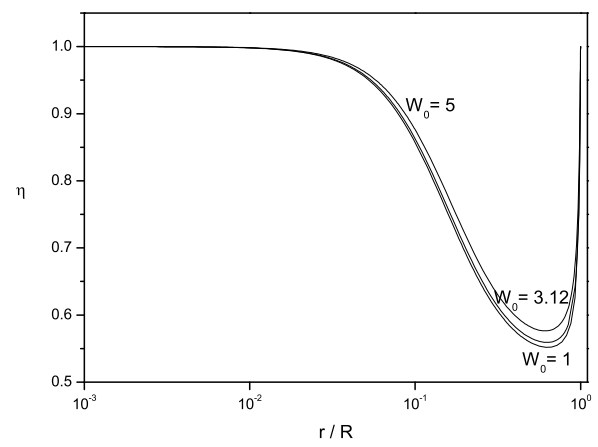

FIGURE 9. Same as in Fig. 6 , for $l=1, a=10^{-3}, \beta=10^{-5}$ and $W_{0}=1 ; 3.12 ; 5$.

$$
\eta=\frac{1+\frac{4}{5}\left(\frac{r}{r_{a}}\right)^{2} \frac{I_{2}}{I_{1}}}{1+\frac{8}{5}\left(\frac{r}{r_{a}}\right)^{2} \frac{I_{2}}{I_{1}}}
$$

where

$$
\begin{aligned}
& I_{1}=\left(\frac{\beta}{1-\beta W}\right)^{5 / 2} \int_{0}^{W} e^{W-x}\left(1+\frac{\beta x / 2}{1-\beta W}\right)^{3 / 2} x^{3 / 2} d x, \\
& I_{2}=\left(\frac{\beta}{1-\beta W}\right)^{7 / 2} \int_{0}^{W} e^{W-x}\left(1+\frac{\beta x / 2}{1-\beta W}\right)^{5 / 2} x^{5 / 2} d x .
\end{aligned}
$$




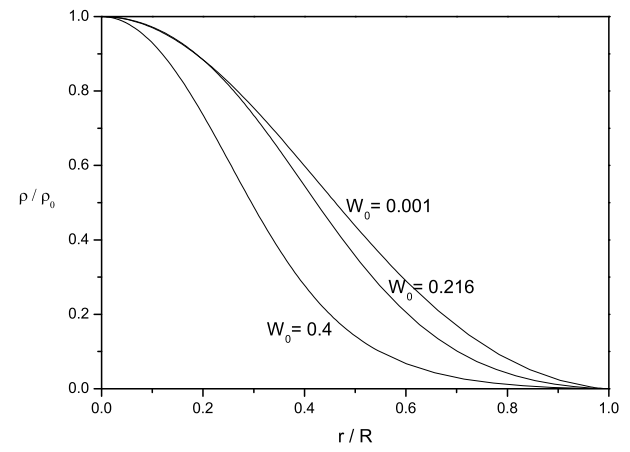

FIGURE 10. Relative density $\rho / \rho_{0}$ as a function of the relative radius $r / R$ along the cluster, for an index $l=1$. The values of the parameters are: $a=10^{-1}, \beta=1$ and $W_{0}=0.001 ; 0.216 ; 0.4$.

At the edge of the cluster, where $r \rightarrow R, I_{2} / I_{1} \rightarrow 0$ so that $\eta \rightarrow 1$. At large anisotropy, when $a$ is smaller, the terms with $I_{2} / I_{1}$ in Eq. (23) are much larger than 1 and the equilibrium configuration reaches the maximal anisotropy corresponding to $\eta_{m}=0.5$. This is the same result obtained in Newtonian regime (see Figs. 8-9).

\section{DENSITY PROFILES OF THE EQUILIBRIUM CONFIGURATIONS}

It is interesting to note that density profiles of equilibrium configurations, described by the distribution function (3), have an increasing behavior in the central region, due to the presence of sufficiently large level of anisotropy, and therefore the maximum density is achieved out of the center. Thus, also in relativistic regime, the existence of "hollow" configurations is clearly a feature of anisotropy and confirms the results obtained by Ralston \& Smith [22] with a different anisotropic distribution function. In Figs. 10-15 we have represented the behavior of density in clusters with different values of $a, \beta$ and $W_{0}$, for an index $l=1$. The triads of values of $W_{0}$ in such figures are chosen with the same criterion considered in Figs. 6-9.

At very large level of anisotropy, the central density may be several orders of magnitude smaller than the maximum value and the maximal density is situated far from the central region of the configuration, especially for decreasing values of $W_{0}$ (see, in particular, Figs. 12 and 15). Moreover, for $a \rightarrow 0$, the cluster is approaching a structure of a thick shell. This situation becomes different at larger values of the anisotropy parameter $a$, when the configuration begins to be more close to the isotropic one: in these cases the maxima of density disappear and the profiles recover the usual monotonic decreasing behavior from the center to the boundary of the equilibrium configuration.

The presence of the maxima of density in a region different from the center of the configuration is clearly related with behavior of the parameter $\eta$ as a function of the 


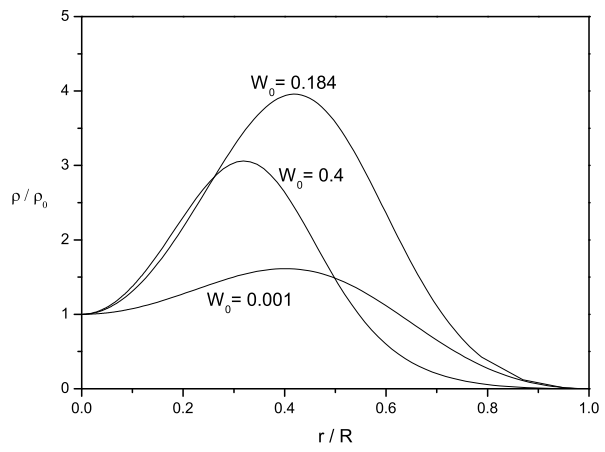

FIGURE 11. Same as in Fig. 10 , for $l=1, a=10^{-2}, \beta=1$ and $W_{0}=0.001 ; 0.184 ; 0.4$.

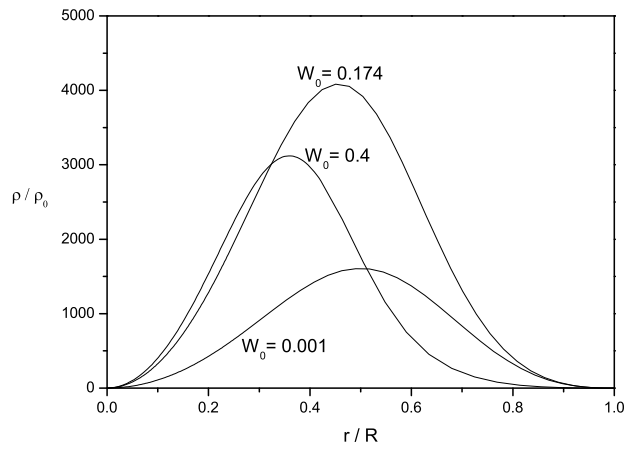

FIGURE 12. Same as in Fig. 10 , for $l=1, a=10^{-5}, \beta=1$ and $W_{0}=0.001 ; 0.174 ; 0.4$. Due to large scale used in the values of $\rho / \rho_{0}$, it seems that the relative density approaches to zero at the center of the configuration, but the actual value is 1 .

relative radius $r / R$ previously discussed. It seems, in fact, that models with $\eta \geq 0.6$ all over the configuration cannot have an "hollow" structure if $\beta=10^{-5}$. For more relativistic models $(\beta=1)$ the critical value of $\eta$ increases to $0.7 \div 0.8$, allowing to obtain hollow configurations at lower levels of anisotropy (see, in particular, Fig. 11).

The results concerning the configurations of Figs. 10-15 are summarized in Table 1, where we have represented the relative $r_{m} / R$ and nondimensional $\tilde{r}_{m}$ radius corresponding to the maximum density for different values of $a, \beta$ and $W_{0}$. 


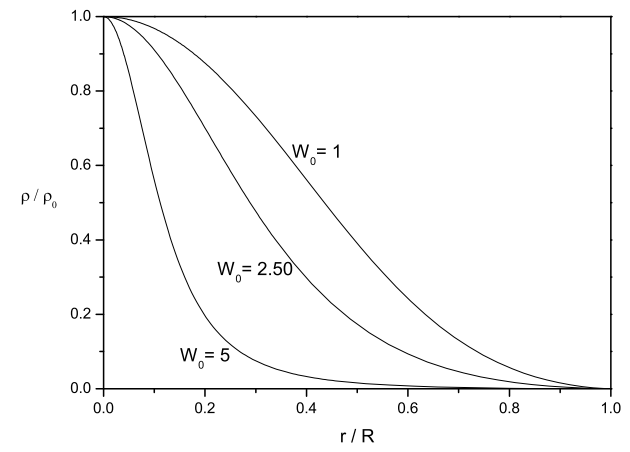

FIGURE 13. Same as in Fig. 10, for $l=1, a=10^{-2}, \beta=10^{-5}$ and $W_{0}=1 ; 2.50 ; 5$.

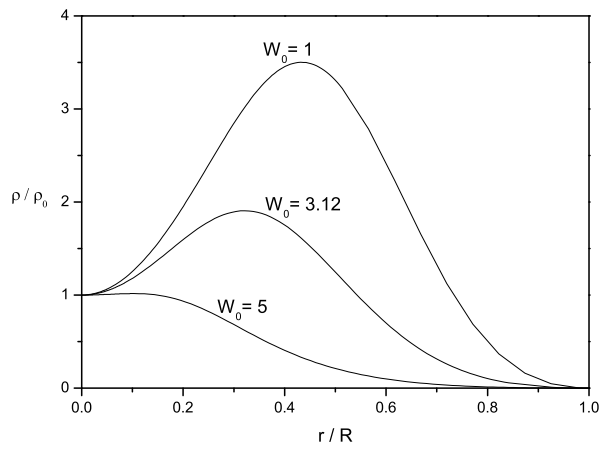

FIGURE 14. Same as in Fig. 10 , for $l=1, a=10^{-3}, \beta=10^{-5}$ and $W_{0}=1 ; 3.12 ; 5$.

\section{STABILITY ANALYSIS FOR ISOTROPIC RELATIVISTIC CLUSTERS}

To analyze the stability of relativistic isotropic clusters we need to calculate the specific binding energy of the equilibrium models. We have calculated two families of curves, which characterize dynamical and thermodynamical stability of relativistic clusters with different cutoff parameters. The curves $E_{b}\left(\rho_{0}\right)$ of specific binding energy at constant temperature $T$ (Fig. 16) characterize the thermodynamical stability, while the curves $E_{b}\left(\rho_{0}\right)$ at constant $W_{0}$ (Fig. 17) give information about dynamical stability of the cluster (Bisnovatyi-Kogan \& Merafina [5]). Relativistic expression of specific binding energy is $E_{b}=(N m-M) / N m$, where $N$ is the total number of stars in the cluster given by 


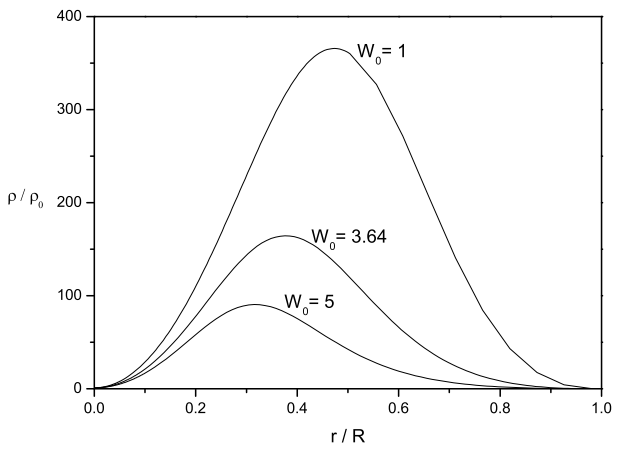

FIGURE 15. Same as in Fig. 10, for $l=1, a=10^{-5}, \beta=10^{-5}$ and $W_{0}=1 ; 3.64$; 5. Like in Fig. 12, the actual value of $\rho / \rho_{0}$ at the center of the configuration is 1 .

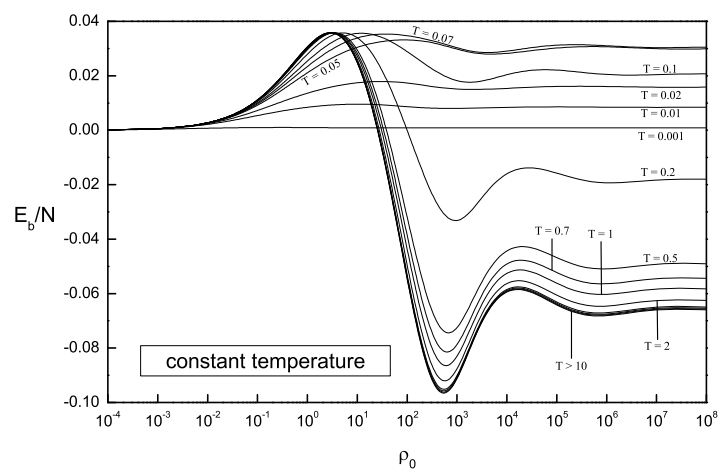

FIGURE 16. Specific binding energy $E_{b} / N$ of equilibrium configurations in clusters with a cutoff as a function of central density $\rho_{0}$ for different values of temperature $T$. Each extremum corresponds to appearance of new thermodynamically unstable modes.

$$
N=4 \pi \int_{0}^{R} \frac{n r^{2} d r}{\sqrt{1-2 G M_{r} / r c^{2}}}
$$

and $m$ is the mass of a single star (all stars have the same mass). The number density $n$ is expressed as usual by integral in momentum space.

The temperature is increasing along each curve in Fig. 17, tending to a finite constant value for large values of central density $\rho_{0}$. The loss of stability, characterized by the first maximum, takes place only for $W_{0} \leq 15.5$. In correspondence of this critical value $W_{0} \simeq 15.5$, the temperature, at large $\rho_{0}$, reaches a limiting value (asymptotic) 
TABLE 1. Some numerical characteristics of the anisotropic clusters with different values of $a, W_{0}$ and $\beta$ : nondimensional radius of the configuration $\tilde{R}$, nondimensional radius of maximal density $\tilde{r}_{m}$, relative radius of maximal density $r_{m} / R$, ratio of the maximal to central density $\rho_{m} / \rho_{0}$. The value of the index of the distribution function is $l=1$.

\begin{tabular}{|c|c|c|c|c|c|c|}
\hline $\mathrm{a}$ & $W_{0}$ & $\beta$ & $\tilde{R}$ & $\tilde{r}_{m}$ & $r_{m} / R$ & $\rho_{m} / \rho_{0}$ \\
\hline $10^{-5}$ & 1 & $10^{-5}$ & $1.98 \cdot 10^{-1}$ & $9.40 \cdot 10^{-2}$ & $4.74 \cdot 10^{-1}$ & $3.66 \cdot 10^{2}$ \\
$10^{-5}$ & 3.64 & $10^{-5}$ & $1.09 \cdot 10^{-1}$ & $4.10 \cdot 10^{-2}$ & $3.76 \cdot 10^{-1}$ & $1.64 \cdot 10^{2}$ \\
$10^{-5}$ & 5 & $10^{-5}$ & $9.20 \cdot 10^{-2}$ & $2.90 \cdot 10^{-2}$ & $3.15 \cdot 10^{-1}$ & $9.05 \cdot 10^{1}$ \\
\hline $10^{-5}$ & 0.001 & 1 & $3.67 \cdot 10^{-2}$ & $1.80 \cdot 10^{-2}$ & $4.90 \cdot 10^{-1}$ & $1.61 \cdot 10^{3}$ \\
$10^{-5}$ & 0.174 & 1 & $4.19 \cdot 10^{-3}$ & $1.89 \cdot 10^{-3}$ & $4.51 \cdot 10^{-1}$ & $4.08 \cdot 10^{3}$ \\
$10^{-5}$ & 0.4 & 1 & $2.37 \cdot 10^{-3}$ & $8.70 \cdot 10^{-4}$ & $3.67 \cdot 10^{-1}$ & $3.10 \cdot 10^{3}$ \\
\hline $10^{-3}$ & 1 & $10^{-5}$ & $1.95 \cdot 10^{0}$ & $8.40 \cdot 10^{-1}$ & $4.32 \cdot 10^{-1}$ & $3.50 \cdot 10^{0}$ \\
$10^{-3}$ & 3.12 & $10^{-5}$ & $1.18 \cdot 10^{0}$ & $3.80 \cdot 10^{-1}$ & $3.21 \cdot 10^{-1}$ & $1.91 \cdot 10^{0}$ \\
$10^{-3}$ & 5 & $10^{-5}$ & $9.75 \cdot 10^{-1}$ & $9.90 \cdot 10^{-2}$ & $1.02 \cdot 10^{-1}$ & $1.02 \cdot 10^{0}$ \\
\hline $10^{-3}$ & 0.001 & 1 & $3.65 \cdot 10^{-1}$ & $1.80 \cdot 10^{-1}$ & $4.93 \cdot 10^{-1}$ & $1.59 \cdot 10^{1}$ \\
$10^{-3}$ & 0.176 & 1 & $4.16 \cdot 10^{-2}$ & $1.90 \cdot 10^{-2}$ & $4.57 \cdot 10^{-1}$ & $4.07 \cdot 10^{1}$ \\
$10^{-3}$ & 0.4 & 1 & $2.38 \cdot 10^{-2}$ & $8.65 \cdot 10^{-3}$ & $3.64 \cdot 10^{-1}$ & $3.09 \cdot 10^{1}$ \\
\hline $10^{-2}$ & 1 & $10^{-5}$ & $4.38 \cdot 10^{0}$ & 0 & 0 & 1 \\
$10^{-2}$ & 2.50 & $10^{-5}$ & $2.96 \cdot 10^{0}$ & 0 & 0 & 1 \\
$10^{-2}$ & 5 & $10^{-5}$ & $2.13 \cdot 10^{0}$ & 0 & 0 & 1 \\
\hline $10^{-2}$ & 0.001 & 1 & $1.07 \cdot 10^{0}$ & $4.30 \cdot 10^{-1}$ & $4.01 \cdot 10^{-1}$ & $1.61 \cdot 10^{0}$ \\
$10^{-2}$ & 0.184 & 1 & $1.27 \cdot 10^{-1}$ & $5.40 \cdot 10^{-2}$ & $4.26 \cdot 10^{-1}$ & $3.98 \cdot 10^{0}$ \\
$10^{-2}$ & 0.4 & 1 & $7.53 \cdot 10^{-2}$ & $2.50 \cdot 10^{-2}$ & $3.32 \cdot 10^{-1}$ & $3.09 \cdot 10^{0}$ \\
\hline $10^{-1}$ & 1 & $10^{-5}$ & $4.74 \cdot 10^{0}$ & 0 & 0 & 1 \\
$10^{-1}$ & 2.48 & $10^{-5}$ & $3.20 \cdot 10^{0}$ & 0 & 0 & 1 \\
$10^{-1}$ & 5 & $10^{-5}$ & $2.23 \cdot 10^{0}$ & 0 & 0 & 1 \\
\hline $10^{-1}$ & 0.001 & 1 & $1.66 \cdot 10^{0}$ & 0 & 0 & 1 \\
$10^{-1}$ & 0.216 & 1 & $2.77 \cdot 10^{-1}$ & 0 & 0 & 1 \\
$10^{-1}$ & 0.4 & 1 & $1.84 \cdot 10^{-1}$ & 0 & 0 & 1 \\
\hline & & & & & \\
\hline
\end{tabular}

$T_{a}=0.0635$. This means that no dynamic instabilities are present for $T \leq 0.06$. At $W_{0}=16$, for example, the limiting temperature is equal to $T_{a}=0.597$ and specific binding energy $E_{b}\left(\rho_{0}\right)$ increases monotonously until the asymptotic value $E_{b, a}=0.0312$ (Bisnovatyi-Kogan \& Merafina [5]).

At large $\rho_{0}$, for models with very large central redshift $z_{c}$, there is an asymptotic value $E_{b, a}$ of specific binding energy for each value of $W_{0}$. Plotting the function $E_{b, a}\left(W_{0}\right)$ from Fig. 17 we obtain a more precise boundary of the dynamical stability $W_{0, a}=15.8$. Due to monotonic dependence of asymptotic (at large $\rho_{0}$ ) values of limiting temperature $T_{a}$ on the parameter $W_{0}$, similar curve $E_{b, a}\left(T_{a}\right)$ from Fig. 16 shows the appearance of dynamically unstable clusters at $T_{a} \geq 0.06$ (Bisnovatyi-Kogan \& Merafina [5]). The limiting curves of specific binding energy $E_{b, a}\left(W_{0}\right)$ and $E_{b, a}\left(T_{a}\right)$ are represented in Figs. 18a and $18 \mathrm{~b}$ respectively. A summary of numerical results on dynamical and thermodynamical stability analysis is given in Figs. 19a and 19b, where different regions are represented in the planes $\left(T, \rho_{0}\right)$ and $\left(T, z_{c}\right)$, respectively. The results plotted in the 


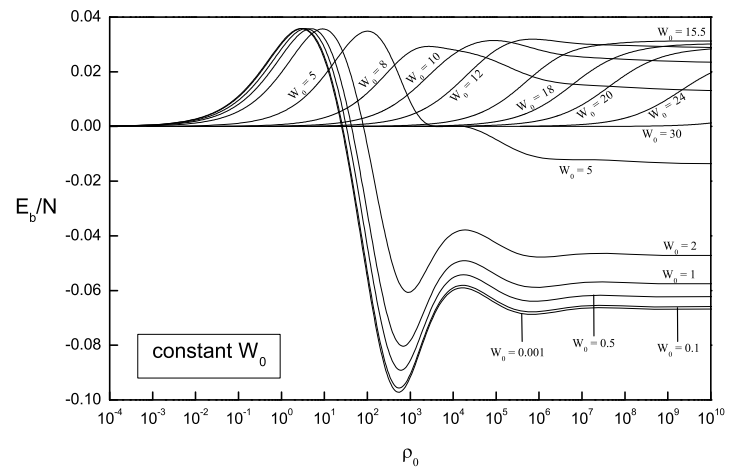

FIGURE 17. Specific binding energy $E_{b} / N$ of equilibrium configurations in clusters with a cutoff as a function of central density $\rho_{0}$ for different values of parameter $W_{0}$. First maxima, corresponding to loss of dynamical stability, are present only on curves with $W_{0} \leq 15.5$. Each extremum corresponds to appearance of new dynamically unstable modes.
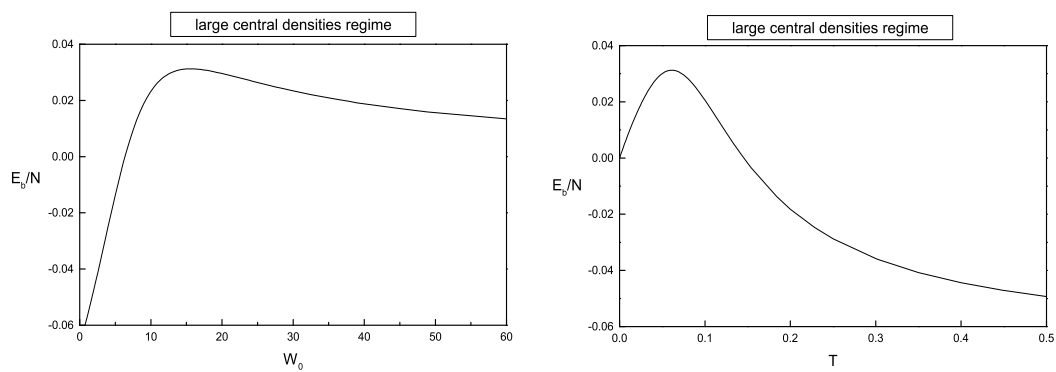

FIGURE 18. Specific binding energy $E_{b} / N$ of equilibrium configurations in clusters with a cutoff for very large central densities $\rho_{0}$ and central redshifts $z_{c}$ as a function of $W_{0}$ (Fig. 18a, left side) and $T_{a}$ (Fig. 18b, right side). The maximum, indicating the loss of dynamical stability, corresponds to $W_{0}=15.8$ and $T \simeq 0.06$, respectively. The limiting value of binding energy is $E_{b, a}=0.0312$.

plane $\left(T, z_{c}\right)$ are analogous to ones of the work of Merafina [18]. It is important to stress the coincidence of boundaries between dynamically and thermodynamically stable and unstable configurations at large temperatures. Using approximate criteria of dynamical stability (see BKMRV93 and BKMRV98) we cannot definitely judge if these boundaries coincide exactly or there is a small difference between them, however the behavior of specific binding energy $E_{b} / N$ let us enough confidence in this result. 

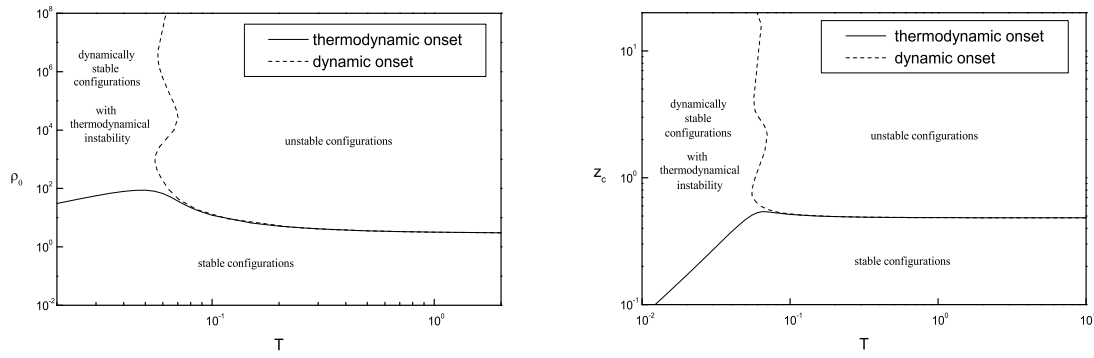

FIGURE 19. Regions of dynamical and thermodynamical stability and instability in the plane $\left(T, \rho_{0}\right)$ (Fig. 19a, left side) and in the plane $\left(T, z_{c}\right)$ analogous to results of Merafina in 1999 (Fig. 19b, right side)

\section{CONCLUSIONS}

We have constructed models of relativistic anisotropic clusters with a distribution function which generalize isotropic models with quasi-equilibrium Maxwellian distribution function with the energy cutoff. The presence of anisotropy leads to density profiles which can exhibit a maximum laying out of the center. This particular feature, called "hollowness", is mainly present in more relativistic configurations and for smaller values of the anisotropy parameter $a$ corresponding to higher levels of anisotropy. Similar kind of anisotropy is created around supermassive black holes due to the existence of a loss cone. Selfgravitation of the cluster is important when the mass of the bulge is comparable to the black hole mass.

By analyzing the behavior of the mass of the equilibrium configurations as a function of the central density, we have seen that the effect of the presence of anisotropy leads to a general decreasing of equilibrium mass at fixed values of the parameter $\beta$. The masses of the equilibrium configurations at increasing values of the central density also show a non monotonic behavior for fixed values of the parameters $\beta$ and $a$. It was shown by Antonov [1] and Lynden-Bell \& Wood [17] that the first maximum on the curve $M\left(\rho_{0}\right)$ for a Newtonian cluster in a box corresponds to loss of the thermodynamic stability of the cluster and the beginning of its rapid contraction. In the paper of Bisnovatyi-Kogan \& Merafina [5] it has been concluded that the behavior of a cluster with a cutoff is very similar to the one in a box, both qualitatively and quantitatively. This result was also extended to relativistic regime. However, analyzing thermodynamic stability in presence of anisotropy may be difficult, being the cluster out of local thermodynamic equilibrium. Nevertheless, conclusions about dynamical and thermodynamical stability of relativistic clusters in presence of anisotropy can be still drawn and systematically discussed by defining specific criteria. These topics may be object of future investigations. 


\section{REFERENCES}

1. Antonov, V.A. 1962, Vest. Leningr. Gos. Univ., 7, 135

2. Bisnovatyi-Kogan, G.S. 1978, Soviet Ast. Lett., 4, 69

3. Bisnovatyi-Kogan, G.S., Merafina, M., Ruffini, R., Vesperini, E. 1993, ApJ, 414, 187 (BKMRV93)

4. Bisnovatyi-Kogan, G.S., Merafina, M., Ruffini, R., Vesperini, E. 1998, ApJ, 500, 217 (BKMRV98)

5. Bisnovatyi-Kogan, G.S., Merafina, M. 2006, ApJ, 653, 1445

6. Bisnovatyi-Kogan, G.S., Merafina, M., Vaccarelli, M.R. 2009, ApJ, 703, 628

7. Bisnovatyi-Kogan, G.S., Merafina, M., Vaccarelli, M.R. 2009, ApJ, submitted

8. Bisnovatyi-Kogan, G.S., Zel'dovich, Ya.B. 1969, Astrofizika, 5, 223

9. Bisnovatyi-Kogan, G.S., Zel'dovich, Ya.B. 1969, Astrofizika, 5, 425

10. Bronstein, I.M., Semendyaev, K.A. 1985, Handbook of Mathematics (New York: Van Nostrand)

11. Einstein, A. 1939, Ann. Math., 40, 922

12. Gürkan, M.A., Freitag, M., Rasio, F.A. 2004, ApJ, 604, 632

13. Ingrosso, G., Merafina, M., Ruffini, R., Strafella, F. 1992, A\&A, 258, 223

14. Ipser, J.R. 1969, ApJ, 158, 17

15. Ipser, J.R. 1980, ApJ, 238, 1101

16. Landau, L.D., Lifshitz, E.M. 1962, The Classical Theory of Fields (London: Pergamon Press)

17. Lynden-Bell, D., Wood, R. 1968, MNRAS, 138, 495

18. Merafina, M. 1999, Odessa Astron. Publ., 12, 210

19. Merafina, M., Ruffini, R. 1989, A\&A, 221, 4

20. Merafina, M., Ruffini, R. 1990, A\&A, 227, 415

21. Rasio, F.A., Shapiro, S.L., Teukolsky, S.A. 1989, ApJ, 336, L63

22. Ralston, J.P., Smith, L.L. 1991, ApJ, 367, 54

23. Suffern, K.G., Fackerell, E.D. 1976, ApJ, 203, 477

24. Zel'dovich, Ya.B., Podurets, M.A. 1965, AZh, 42, 963 